\title{
Abbildungsanalyse rekonstruktiver und ersetzender Therapieverfahren in der Orthopädie und Unfallchirurgie im G-DRG-System 2016
}

\author{
Moritz Freistühler, Dominik Franz
}

\section{Zusammenfassung}

Bei der täglichen Versorgung von Verletzungen und insbesondere bei der Frakturversorgung stellt sich an einigen Lokalisationen die Frage, ob ein rekonstruktives oder ein ersetzendes Therapieverfahren gewählt werden sollte. Besonders bei der Frakturversorgung von älteren Patienten mit vielen Begleiterkrankungen oder degenerativen Vorschädigungen sind osteosynthetische Versorgungen auch aufgrund der oft verminderten Knochenqualität nur schwer und unter erheblichem Aufwand durchführbar. Die Operationen finden oft nicht elektiv statt, sondern werden im Rahmen der täglichen Notfallversorgung im Nachtdienst mit reduziertem Personal im Vergleich zur Regelarbeitszeit durchgeführt. Auch Unterschiede in der postoperativen Behandlung mit Teilbelastung der betroffenen Extremität nach osteosynthetischer Versorgung spielen bei der Indikationsstellung oftmals eine entscheidende Rolle. Daher ist auch der endoprothetische Ersatz eines Gelenks bei der Versorgung von Frakturen je nach individueller Fallkonstellation zu erwägen. Unabhängig von diesen klinischen Fragestellungen stellt sich im DRG-Zeitalter und bei zunehmendem wirtschaftlichem Druck im Gesundheitswesen auch die Frage der sachgerechten Vergütung für die erbrachten Leistungen. Der Vergleich der Abbildung von osteosyntheti- schen und endoprothetischen Operationsverfahren bei der Frakturversorgung an 4 verschiedenen Lokalisationen des Körpers zeigt, dass es im G-DRG-System 2016 eine deutlich differenzierte Darstellung der osteosynthetischen Versorgung bei Frakturen gibt. Die endoprothetischen Leistungen werden meist unabhängig von der Ursache (Fraktur vs. degenerative Erkrankung als Hauptdiagnose) einer G-DRG zugeordnet. Sie werden zudem in ökonomisch höher bewertete G-DRGs eingruppiert und erbringen somit auch einen höheren Erlös. Bei Berücksichtigung der oftmals höheren Implantatkostenanteile sowie der längeren stationären Verweildauern dieser G-DRGs relativiert sich dieser höhere Erlös jedoch an einigen Stellen deutlich. An anderen Lokalisationen und in bestimmten Konstellationen finden sich jedoch deutliche Unterschiede, woraus sich u.U. auch systembedingte Fehlanreize ergeben können.

\section{Analysis of Reconstructive and Replacing Surgical Techniques in Orthopedics and Trauma Surgery in the German DRG-System 2016}

In the daily treatment of injuries and in particular in the treatment of fractures at some locations the question arises whether to do a reconstructive surgery or an arthroplasty. Especially in the treatment of elderly patients with multiple comorbidities and degenerative predamage osteosynthetic operation techniques are often even more difficult because of a poor bone quality. The operations are often done in the nighttime during the emergency care with a reduced headcount instead of being done with good preparation during the day. Differences in postoperative treatment with partial weight bearing of the affected limb after osteosynthesis play a decisive role in the selection of the right type of treatment. Therefore the arthroplasty is considered to be a good type of treatment in some cases and at some locations. Despite of these clinical questions there is a need of proper reimbursement in times of G-DRGs and increasing economic pressure in the German health care system. The comparison of osteosynthetic and endoprosthetic surgery in fracture treatment at different locations of the body shows a clearly differentiated illustration of osteosynthetic care for fractures in the current G-DRG-System 2016. The arthroplasty is assigned independently of the main diagnosis to a G-DRG. They are also grouped into economically higher rated DRGs and thus also provide a higher reimbursement. This higher return is reduced by considering the implant costs and a prolonged length of stay which are included in each G-DRG. Nevertheless there are differences at some locations and in some constellations even after deduction of implant costs.

\section{Einleitung}

In der stationären Krankenversorgung in Deutschland wird seit dem Jahr 2004 ein fallpauschaliertes Entgeltsystem -

OP-JOURNAL 2016; 32: 247-253

(c) Georg Thieme Verlag KG Stuttgart · New York DOI http://dx.doi.org/10.1055/s-0036-1595901
G-DRG-System - eingesetzt. Die mit der Einführung des G-DRG-Systems verbundenen wesentlichen Ziele waren die Berücksichtigung der Leistungsorientierung, die Therapiekomplexität und die Komorbidität der Patienten. Die medizinische Versorgung, die mit einem hohen Ressourcenverbrauch einhergeht, soll höher vergütet werden als Therapien mit geringerem Ressourcenverbrauch.
Darüber hinaus sollte die Transparenz von stationären Krankenhausleistungen und deren Kosten gesteigert werden.

Grundlagen für die Zuordnung des einzelnen Behandlungsfalls zu einer G-DRGFallpauschale sind die routinemäßig dokumentierten klinischen Patientendaten. Die fallindividuellen Diagnosen und durchgeführten medizinischen Maßnah- 
men werden nach den vom Deutschen Institut für Medizinische Dokumentation und Information (DIMDI) herausgegebenen Klassifikationssystemen erfasst. Für Diagnosen gilt die jeweils aktuelle Version des ICD-10-GM-Katalogs [4]. Während des Aufenthalts durchgeführte diagnostische sowie therapeutische Prozeduren werden nach dem aktuellen deutschen Operationen- und Prozedurenschlüssel (OPS) verschlüsselt [3].

Um eine deutschlandweit möglichst einheitliche Kodierung zu gewährleiten, werden vom Institut für das Entgeltsystem im Krankenhaus (InEK) in Zusammenarbeit mit der Deutschen Krankenhausgesellschaft (DKG), dem GKV-Spitzenverband sowie dem Verband der Privaten Krankenversicherungen (PKV) jährlich aktualisierte und verbindliche Kodierrichtlinien veröffentlicht [5]. Das InEK ist als deutsches DRG-Institut damit beauftragt, das G-DRG-System zu pflegen und weiterzuentwickeln. Es definiert den Gruppierungsalgorithmus zu den einzelnen G-DRGs und kalkuliert deren Bewertungen.

Je nach kodierten Diagnosen (differenziert nach Hauptdiagnose und Nebendiagnosen), Prozeduren und Patientendaten, wie z.B. dem Patientenalter und weiteren Stammdaten, werden die Behandlungsfälle in bestimmte G-DRGs gruppiert. Die in der Systemversion 2016 ca. 1200 verschiedenen DRGs werden durch einen 4-stelligen alphanumerischen Kode beschrieben [1]. Die 1. Stelle bildet ein Buchstabe und steht für eine der 23 Hauptdiagnosegruppen (MDC: Major Diagnostic Category) des Fallpauschalenkatalogs, danach folgt eine 2stellige Zahl. Beide Parameter bilden die sog. Basis-DRG. An der 4. Stelle steht wiederum ein Buchstabe, der die BasisDRG in weitere Untergruppen (DRGSplits) differenziert. Die verschiedenen DRG-Splits repräsentieren eine unterschiedliche ökonomische Fallschwere in Bezug auf die Basis-DRG. Hierzu werden, je nach Höhe des kalkulierten Ressourcenverbrauchs, einzelne Diagnose- oder Prozedurengruppen definiert, deren Kombination die Zuteilung in den jeweiligen Split zur Folge hat. Hierbei spielt u.U. auch die Anzahl und Art der Nebendiagnosen in Bezug zur Hauptdiagnose eine besondere Rolle, da diese Aufschlüsse über Komorbiditäten und Komplikationen der Patienten geben können. Nebendiagnosen können unter Berücksichtigung des patientenbezogenen Gesamtschweregrads (PCCL: Patient Clinical
Complexity Level) bei der G-DRG-Zuordnung Berücksichtigung finden. Der PCCL ist in 7 Stufen (0-6) unterteilt. Je höher die Stufe, desto gravierender die Komorbidität.

Jede G-DRG ist mit einer Bewertungsrelation (BWR) bewertet, die durch Multiplikation mit dem jeweiligen Basisfallwert des Bundeslands den Erlös in Euro für die Krankenhäuser dieses Bundeslands bestimmt. Für die Erlösermittlungen dieser Arbeit wurde der Bundesbasisfallwert 2016 in Höhe von 3311,98€ verwendet. Die Höhe der Bewertungsrelation wird alljährlich aus den Kostendaten von ca. 250 sog. Kalkulationskrankenhäusern vom InEK kalkuliert. Die jeweils aktuelle Systemversion basiert auf den Kosten- und Abrechnungsdaten des Vorjahrs und gilt für das Folgejahr. So wird z.B. aus den Daten des Jahres 2014 das G-DRG-System für das Jahr 2016 erstellt.

Durch diese jährlich stattfindenden Analysen des InEK ergeben sich jedes Jahr auch Anpassungen, Neubewertungen oder Neustrukturierungen von G-DRGs im Bereich der Orthopädie und Unfallchirurgie. Diese Anpassungen werden auch durch die Deutsche Gesellschaft für Orthopädie und Unfallchirurgie vorangetrieben, die im Rahmen des jährlichen Vorschlagsverfahrens Anregungen zur Analyse von Fallgruppen an das InEK gibt [7].

Dieser Artikel soll die aktuelle Abbildung von rekonstruktiven Behandlungen im Vergleich zu ersetzenden Verfahren an ausgewählten Körperlokalisationen im Bereich der Orthopädie und Unfallchirurgie im G-DRG-System 2016 darstellen und analysieren. Hierzu wurden typische Frakturdiagnosen nach der ICD-Klassifikation klassifiziert und jeweils die rekonstruktiven und die ersetzenden operativen Standardtherapieverfahren anhand der OPS-Klassifikation verschlüsselt und die Gruppierungsergebnisse verglichen. Hierbei wurden auch die Auswirkungen von Komorbiditäten und Komplikationen anhand des PCCL auf die Zuteilung zu einer bestimmten G-DRG berücksichtigt. Mehrfachverletzungen und längere intensivmedizinische Aufenthalte wurden bei den ausgewählten Beispielen nicht berücksichtigt, um die Vergleichbarkeit der Gruppierungsergebnisse zu gewährleisten. Die angegebenen G-DRGErlöse beziehen sich auf Normallieger mit einer tatsächlichen Verweildauer zwischen der unteren und oberen Grenz- verweildauer der jeweiligen G-DRG. Aboder Zuschläge wurden nicht berücksichtigt.

Die bei den einzelnen G-DRGs beschriebenen Implantatkosten sind dem G-DRGReport-Browser 2016 des InEK entnommen [6]. Diese Kosten wurden aus den Bewertungen der einzelnen G-DRGs herausgerechnet, um Vergleiche unabhängig von den Implantatkosten durchführen zu können.

Selbstverständlich ist die Entscheidung, ob ein rekonstruktiver oder ein gelenkersetzender endoprothetischer Eingriff durchgeführt wird, eine medizinische Entscheidung, die dem Operateur vorbehalten ist. Diese Entscheidung sollte aus der Sicht der Autoren unabhängig von ökonomischen Faktoren anhand des individuellen Patientenprofils und des Verletzungsmusters getroffen werden. Das G-DRG-System sollte keinen Anreizen zur Anwendung einer bestimmten Prozedur Vorschub leisten.

\section{Schenkelhalsfraktur}

Eine der häufigsten unfallchirurgischen Diagnosen ist die Schenkelhalsfraktur. Diese wird in der ICD-10-GM mit dem Kode S72.0* beschrieben. Die 5. Stelle des Kodes beziffert die exakte Frakturlokalisation, diese hat jedoch keine Auswirkung auf das spätere Gruppierungsergebnis. Die bei dieser Diagnose gängigen Verfahren der Rekonstruktion mit einer dynamischen Hüftschraube (DHS) mit oder ohne Antirotationsschraube wurden mit dem endoprothetischen Gelenkersatz mittels Totalendoprothese oder Duokopfprothese verglichen und der Einfluss von Komorbidität und Komplexität der Patienten anhand der kodierten Nebendiagnosen (PCCL s.o.) auf die G-DRG-Zuteilung berücksichtigt.

Die Rekonstruktion mittels DHS bildet sich über die Basis-DRG I08 „Andere Eingriffe an Hüftgelenk und Femur“ ab, die 8-fach differenziert wird (Split A-H). Welche G-DRG angesteuert wird, hängt hier von der Art der durchgeführten Reposition (geschlossen vs. offen), dem Frakturtyp (einfach vs. mehrfragmentär), dem begleitenden Weichteilschaden und dem PCCL der Patienten ab. Die geschlossene Reposition (OPS 5-790.8e) und die offene Reposition einer einfachen (OPS 5-793.5e) oder mehrfragmentären Fraktur (OPS 5-794.4e) ohne einen relevanten Weichteilschaden (ab Grad III geschlossen und ab Grad II offen) und ohne 




Abb. 1 G-DRG-System 2016: Darstellung der Versorgung einer Schenkelhalsfraktur (HD S72.0*) mittels dynamischer Hüftschraube (DHS). Der Unterschied der DRGS I08D und I08E liegt bei gleicher BWR in den unterschiedlichen Grenzverweildauern und den evtl. damit verbundenen Zu- bzw. Abschlägen; ORIF: Open Reduction and Internal Fixation; CRIF: Closed Reduction and Internal Fixation; rW: gruppierungsrelevanter Weichteilschaden (Grad III geschlossen, ab Grad II offen).

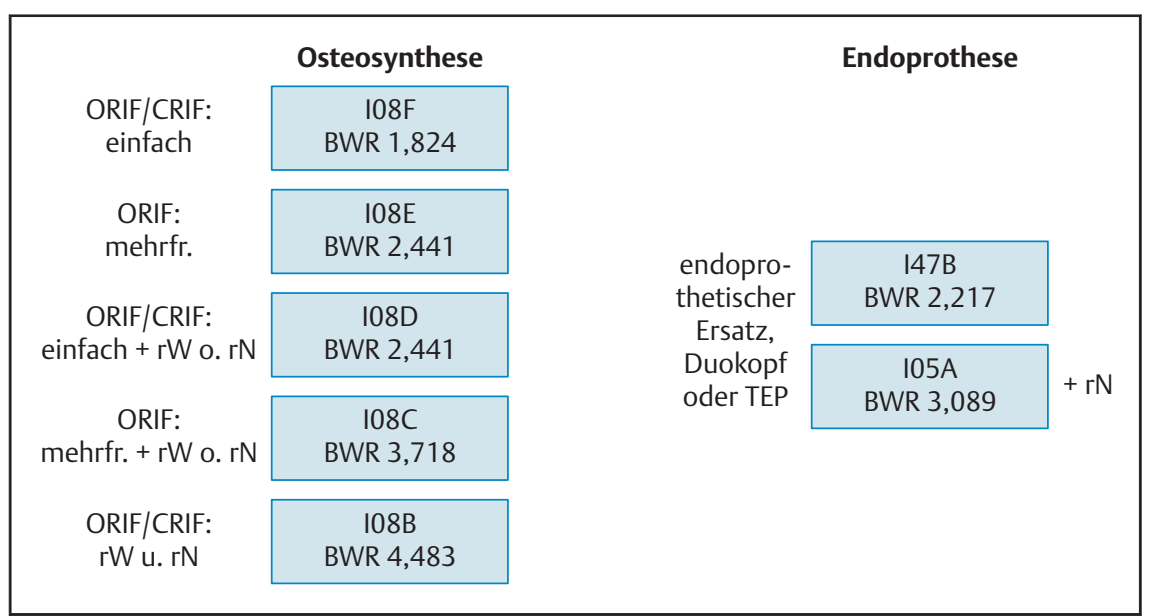

Abb. 2 G-DRG-System 2016: Gegenüberstellung der G-DRG-Zuordnung bei Hauptdiagnose "Schenkelhalsfraktur“ (ICD S72.0*) und Rekonstruktion mittels Osteosynthese oder Gelenkersatz. Therapieverfahren: Einfach: einfache Frakturform mit 2 Fragmenten; Mehrfr.: mehrfragmentäre Frakturform mit mehr als 2 Fragmenten; ORIF: Open Reduction and internal Fixation; CRIF: Closed Reduction and internal Fixation; rW: gruppierungsrelevanter Weichteilschaden (Grad III geschlossen, ab Grad II offen); rN: relevante Nebendiagnosen im Sinne eines PCCL > 3 .

PCCL-relevante Nebendiagnosen mit einer dynamischen Hüftschraube bildet sich über die DRG I08F mit einer BWR von $1,824 \mathrm{ab}$. Dies entspricht unter Berücksichtigung des Bundesbasisfallwerts 2016 (s.o.) einem G-DRG-Erlös von $6041 €$. Eine zusätzlich durchgeführte Schraubenosteosynthese (inkl. Antirotationsschraube) wirkt sich nur bei der offenen Reposition einer mehrfragmentären Fraktur auf die G-DRG-Gruppierung aus und führt in die I08E (BWR 2,441; $8085 €)$. Ein relevanter Weichteilschaden führt, je nach Versorgungsform, ebenfalls zu einer Gruppierung in eine höher bewertete G-DRG (Abb.1). Nebendiag- nosen wirken sich erst ab einer hohen Komorbidität (PCCL > 3) auf das Gruppierungsergebnis aus. Die Abb. 1 stellt die Gruppierungsergebnisse noch einmal übersichtlich dar.

Im Vergleich hierzu führt die gelenkersetzende Therapie mit Implantation einer Duokopfprothese (OPS 5-820.4*, Endstelle: 0 = nicht zementiert, 1 = zementiert) oder einer Totalendoprothese (OPS 5-820.0*, Endstelle: 0 und 1 analog zur Duokopfprothese, 2 = hybrid) jeweils in die G-DRG I47B (BWR 2,217; Erlös $7343 €$ ). Bei einem PCCL von mehr als 3 ergibt sich sowohl bei der Versorgung mittels Duokopf- als auch bei der mit einer Totalendoprothese die DRG I05A (BWR 3,089; Erlös $10231 €$ ). Ein Weichteilschaden hat keinen Einfluss auf das Gruppierungsergebnis.

Beim Vergleich der gelenkersetzenden und gelenkerhaltenden Therapien fällt eine deutlich differenzierte Abbildung im Bereich der gelenkerhaltenden Therapie im G-DRG-System 2016 auf. Hier führt nicht nur eine hohe Komorbidität (PCCL), sondern auch die Art der Fraktur (einfach vs. mehrfragmentär) und der Weichteilschaden $\mathrm{zu}$ einer abgestuften Zuordnung innerhalb der Basis-DRG I08 und somit zu unterschiedlichen G-DRGBewertungen und -erlösen (Abb. 1 und 2). Der „Standardfall“ einer gelenkerhaltenden Therapie mit geschlossener Reposition mittels DHS mit oder ohne Antirotationsschraube und ohne komplizierende Nebendiagnosen $(\mathrm{PCCL}<4)$ ist mit der G-DRG I08F ökonomisch geringer bewertet als der vergleichbare Fall bei gelenkersetzender Therapie und Versorgung mittels Duokopf- oder Totalendoprothese in der G-DRG I47B. Auch die mittleren Verweildauern beider G-DRGs sind vergleichbar. Der Implantatkostenanteil beträgt in der G-DRG I08F 9,1\% und in der I47B 20,1\% [6]. Um diesen Faktor bereinigt beträgt die BWR in der I08F 1,659 und in der I47B 1,77. Dies entspricht einer Differenz von 0,111 BWR und somit $368 €$. Der Einfluss des Weichteilschadens ist theoretisch vorhanden, dürfte jedoch an dieser Lokalisation nur in seltenen Fällen und in Verbindung mit schweren Verletzungsmechanismen relevant werden.

\section{Tibiakopffrakturen}

Im Bereich des Kniegelenks wird die gelenkerhaltende Therapie mit der gelenkersetzenden nach Tibiakopffrakturen verglichen. Die Fraktur des Tibiakopfs wird mit Diagnosen aus S82.1* verschlüsselt. Die letzte Ziffer gibt Auskunft über eine ggf. gleichzeitige Beteiligung der Fibula. Die gelenkerhaltenden Osteosynthesen führen alle in die Basis-DRG I13 und unterscheiden sich je nach durchgeführter Repositionsart (offen vs. geschlossen), nach Art und Anzahl des eingebrachten Osteosynthesematerials sowie anhand eines die Fraktur begleitenden Weichteilschadens.

Die geschlossenen Repositionsformen (OPS 5-790.*k) mit Plattenosteosynthesen (auch winkelstabil) und/oder Schraubenosteosynthesen führen in die G-DRG 


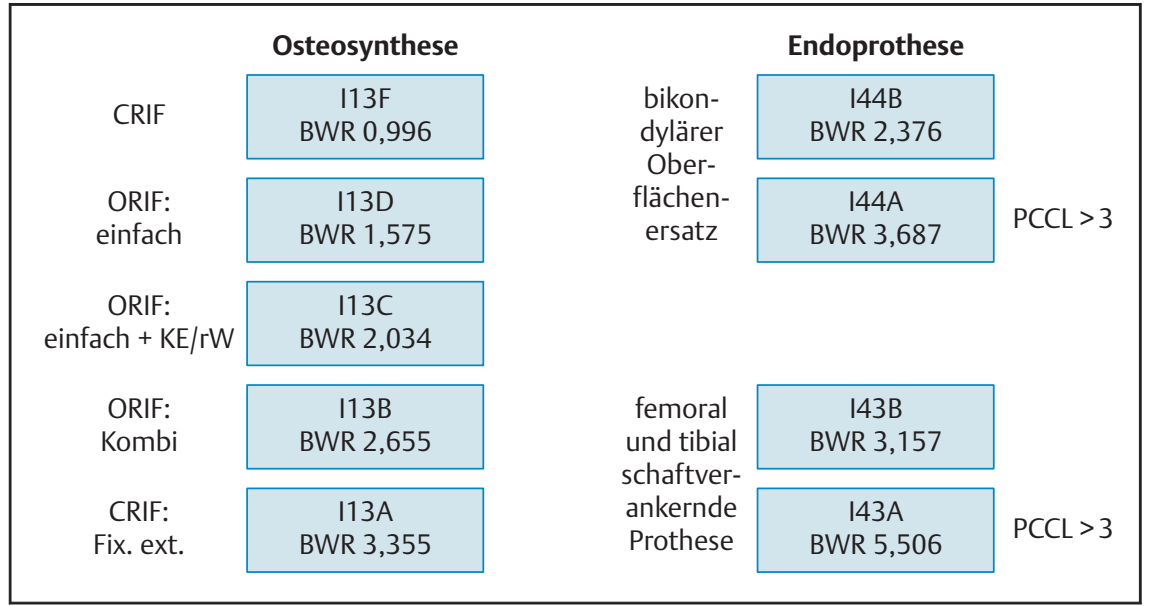

Abb.3 G-DRG-System 2016: Gegenüberstellung der G-DRG-Zuordnung bei Hauptdiagnose „Tibiakopffraktur“ (ICD S82.1*) und Rekonstruktion mittels Osteosynthese oder Gelenkersatz. Therapieverfahren: Fix. ext.: Fixateur externe; Einfach: Osteosynthese mit einem Verfahren, z. B. eine Plattenosteosynthese; Kombi: Osteosynthese mit einer Kombination aus verschiedenen Osteosynthesearten, z.B. eine Plattenosteosynthese und eine Schraubenosteosynthese; KE: keramischer Knochenersatz; rW: relevanter Weichteilschaden.

I13F (BWR 0,996; $3299 €$ ). Die Verwendung von mehr als einer Platte zur Osteosynthese oder eine Kombination aus Platten- und Schraubenosteosynthese hat bei geschlossener Versorgung keine Auswirkungen auf die Gruppierung. Sofern ein relevanter Weichteilschaden (Definition s.o.) vorliegt, erfolgt die $\mathrm{Zu}-$ ordnung zur G-DRG I13D (BWR 1,575; $5216 €)$.

Die offene Reposition und Osteosynthese mit einer Platte (auch winkelstabil) führt unabhängig von der Frakturform in die G-DRG I13D. Ein zusätzlicher relevanter Weichteilschaden oder eine Kombination von Osteosynthesematerialien (z.B. Platten/Schrauben) wird der G-DRG I13C (BWR 2,034; 6737€) zugeordnet. Sofern ein relevanter Weichteilschaden vorliegt, wird die G-DRG I13B (BWR 2,655; $8793 €)$ erreicht. Die additive Verwendung von autologer Spongiosa im Rahmen der Reposition (OPS 5-784.0 k) der Fraktur wirkt sich bei diesen Fallkonstellationen nicht auf die G-DRG-Gruppierung aus.

Allerdings führt die Verwendung eines kortikospongiösen Spans (5-784.1 k) in Kombination mit einer offenen Reposition und einer Osteosynthese in die G-DRG I13B. Sofern z.B. alloplastischer Knochenersatz verwendet wird, wird in gleicher Kombination die G-DRG I13C erreicht. Die geschlossene Reposition mit einem Fixateur externe führt unabhängig von anderen Osteosyntheseformen in die DRG I13A (BWR 3,355; $11112 €)$.
Bei einem endoprothetischen Ersatz des Kniegelenks bei Tibiakopffraktur ergibt sich bei der Implantation eines bikondylären Oberflächenersatzes (Kodierung inklusive Wedges und einseitiger Schaftverankerung über den OPS 5-822.g*, Endstelle analog zur Hüftendoprothetik) die G-DRG I44B (BWR 2,376; $7869 €$ ). Sofern erhebliche Komorbiditäten vorliegen (PCCL > 3) erfolgt die G-DRG-Zuordnung zur I44A (BWR 3,687; 12211€). Sofern eine femoral und tibial schaftverankerte Prothese (OPS 5-822.h*) erforderlich ist, ergibt sich die G-DRG I43B (BWR 3,157; 10456€). Bei einem PCCL $>3$ erfolgt Gruppierung in die G-DRG I43A (BWR 5,506; $18236 €$ ). Ein begleitender Weichteilschaden spielt beim endoprothetischen Ersatz für die DRGGruppierung keine Rolle.

Die Spannweite der G-DRG-Bewertungen beträgt bei der rekonstruktiven Versorgung 0,996-3,355 und bei der endoprothetischen 2,376-5,506. Bereinigt man auch hier die Bewertungsrelationen von den Implantatkostenanteilen und vergleicht die G-DRG I13B (Implantatkostenanteil: 10,9\%) mit der I43B $(27,2 \%)$, die bei den ersetzenden Verfahren für eine aufwendigere prothetische Versorgung mittels tibial und femoral schaftverankerter Endoprothese steht, zeigt sich, dass die bereinigten G-DRGBewertungen mit 2,374 (I13B) und 2,297 (I43B) nur minimal (255€) zugunsten der osteosynthetischen Versorgung abweichen [6]. Bei der Berücksichtigung von Implantatkosten muss ergänzend beachtet werden, dass bei mehrfrag- mentärer Tibiakopffraktur und Implantation einer modularen Prothese ggf. noch ein krankenhausindividuell zu verhandelndes Zusatzentgelt (ZE2016-25, OPS 5-829.k) abgerechnet werden könnte. Hierbei sind jedoch sowohl bez. der knöchernen Defektsituation als auch bei der Art der Prothese besondere Voraussetzungen zu beachten. Die mittleren Verweildauern der DRGs sind mit 12,2 (I13B) und 11,9 (I43B) vergleichbar.

\section{Humeruskopffraktur}

Analog zum Vorgehen bei Schenkelhalsund bei Tibiakopffrakturen erfolgte der Vergleich der rekonstruktiven bzw. der osteosynthetischen Therapie mit dem endoprothetischen Ersatz bei der Behandlung von Humeruskopffrakturen. Die Humeruskopffraktur wird über den ICD S42.2* verschlüsselt, wobei die letzte Ziffer Informationen über die genaue Art und Lokalisation beinhaltet. Eine mehrfragmentäre Humeruskopffraktur (>2 Fragmente) wird bspw. mit S42.21 klassifiziert. Die osteosynthetischen Therapieverfahren führen wie bei der Tibiakopffraktur (s.o.) in die Basis-DRG I13. Daher sind auch bei der Frakturversorgung im Bereich des Humeruskopfs mittels Osteosynthese die geschlossene oder offene Repositionsart, der Weichteilschaden und die Anzahl des eingebrachten Osteosynthesematerials für die GDRG-Gruppierung relevant. Der PCCLWert bleibt bei der Gruppierung unberücksichtigt.

Osteosynthesen mit geschlossener Repositionsform (OPS 5-790.*1) führen unabhängig von der Anzahl der eingebrachten Osteosynthesematerialien in die G-DRG I13F (BWR 0,996; 3299€), in Kombination mit einem relevanten Weichteilschaden (s.o.) in die I13D (BWR 1,575; 5216€). Die offene Reposition und Frakturversorgung bei einfacher Fraktur mittels Schrauben- oder Drahtosteosynthese führt ebenfalls in die G-DRG I13F und die Kombination mit einem relevanten Weichteilschaden in die G-DRG I13D. Die offene Reposition und Frakturversorgung bei mehrfragmentärer Fraktur des proximalen Humerus mittels Schrauben- oder Drahtosteosynthese führt jedoch in die G-DRG I13E (BWR 1,393; 4614€) und die Kombination mit einem relevanten Weichteilschaden in die G-DRG I13C (BWR 2,034; $6737 €)$.

Die offene Reposition und Osteosynthese einer einfachen oder mehrfragmentären 
Humeruskopffraktur mit einer Plattenosteosynthese führt zur Gruppierung in die G-DRG I13D (BWR 1,575; 5216€) und die Kombination mit einem relevanten Weichteilschaden in die DRG I13C (BWR 2,034; 6737€). Bei der Kombination verschiedener Osteosynthesearten zeigt sich, dass eine Kombination aus Platten- und Schraubenosteosynthese (auch mit evtl. zusätzlich eingebrachter Cerclagen- oder Drahtosteosynthese) in die G-DRG I13C führt, die Versorgung mit einer Doppelplattenosteosynthese (auch winkelstabil) führt zu einer Gruppierung in die I13B (BWR 2,655; $8793 €$ ).

Der endoprothetische Ersatz einer (mehrfragmentären) Humeruskopffraktur durch eine Humeruskopfprothese (OPS 5-824.01) sowie auch der komplette Ersatz des Schultergelenks mittels anatomischer oder inverser Prothese (OPS 5-824.2*; 0: anatomisch, 1: invers) führt jeweils unabhängig vom Weichteilschaden oder der Komorbidität der Patienten in die I05B (BWR 2,726; $9028 €)$.

Die differenzierte Abbildung rekonstruktiver Eingriffe zeigt sich auch im Bereich der Versorgung von Humeruskopffrakturen. Demgegenüber bilden sich die ersetzenden Verfahren unabhängig von weiteren Faktoren über eine einzige G-DRG ab (Abb. 4). Der Implantatkostenanteil der G-DRG I05B beträgt 27,5\%, der Anteil der I13D, der für die häufige Frakturversorgung durch Plattenosteosynthese steht, beträgt $10,7 \%$ [6]. Werden die Bewertungsrelationen um die Implantatkostenanteile bereinigt, verbleibt eine Differenz von 0,57. Dies entspricht $1888 €$. Hierbei muss berücksichtigt werden, dass die mittlere Verweildauer der G-DRG I13D 7,6 Tage beträgt. Die der G-DRG I05B ist mit 10,5 Tagen deutlich länger. Die bei Tibiakopffrakturen (s. o.) beschriebenen Regelungen für modulare Endoprothesen (ZE2016-25) gelten auch für den endoprothetischen Ersatz des Schultergelenks.

\section{Radiuskopffraktur}

Die Fraktur des proximalen Radius wird über den ICD-Kode S52.1* verschlüsselt. Die letzte Stelle beschreibt hier erneut die genaue Lokalisation, wobei die Ziffer 1 für den Radiuskopf steht. Hier erfolgt die Abbildung der osteosynthetetischen Versorgung über mehrere Basis-DRGs. Entscheidend bei dieser rekonstruktiven Versorgungsform ist in diesem Fall die Art und Menge des Osteosynthesema-

\begin{tabular}{|c|c|}
\hline & Osteosynthese \\
\hline $\begin{array}{c}\text { CRIF o. } \\
\text { ORIF einfach }\end{array}$ & $\begin{array}{c}\text { I13F } \\
\text { BWR 0,996 }\end{array}$ \\
\hline $\begin{array}{l}\text { ORIF: } \\
\text { mehrfr. }\end{array}$ & $\begin{array}{c}\text { I13E } \\
\text { BWR 1,393 }\end{array}$ \\
\hline $\begin{array}{c}\text { ORIF: Platte o. } \\
\text { CRIF + rW }\end{array}$ & $\begin{array}{c}\text { I13D } \\
\text { BWR 1,575 }\end{array}$ \\
\hline $\begin{array}{l}\text { ORIF: Platte + } \\
\text { andere o. rW }\end{array}$ & $\begin{array}{c}\text { I13C } \\
\text { BWR 2,034 }\end{array}$ \\
\hline $\begin{array}{c}\text { ORIF: } \\
\text { Doppelplatte }\end{array}$ & $\begin{array}{c}\text { I13B } \\
\text { BWR 2,655 }\end{array}$ \\
\hline
\end{tabular}

Endoprothese

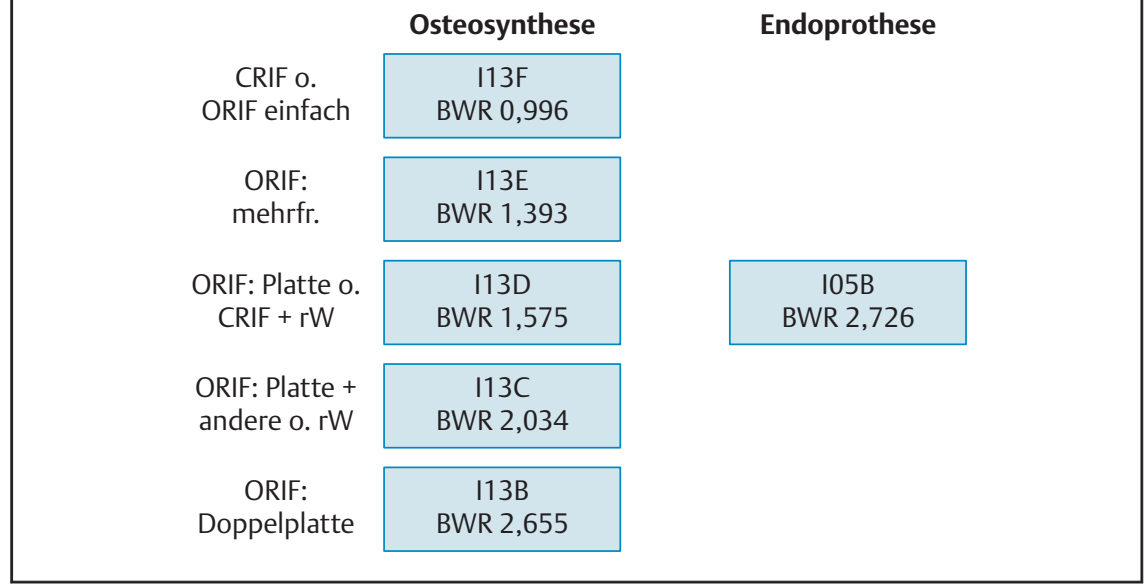

Abb. 4 G-DRG-System 2016: Gegenüberstellung der G-DRG-Zuordnung bei Hauptdiagnose „Fraktur des proximalen Endes des Humerus“ (ICD S42.2*) und Rekonstruktion mittels Osteosynthese oder Gelenkersatz. Therapieverfahren: Einfach: einfache Frakturform mit 2 Fragmenten; Mehrfr.: mehrfragmentäre Frakturform mit mehr als 2 Fragmenten; ORIF: Open Reduction and internal Fixation; CRIF: Closed Reduction and internal Fixation; rW: gruppierungsrelevanter Weichteilschaden (Grad III geschlossen, ab Grad II offen); Doppelplatte: Osteosynthese mit 2 Plattenosteosynthesen; Andere: Osteosyntheseart Draht-, Cerclage- oder Schraubenosteosynthese.

terials sowie die Frakturform (einfach vs. mehrfragmentär). Weichteilschäden oder Komorbiditäten der Patienten (PCCL) sind für die DRG-Zuordnung nicht relevant. Für eine vergleichende Analyse rekonstruktiver und ersetzender Therapieverfahren möchten wir uns im Folgenden auf die Versorgung offener Frakturen fokussieren. Die offene Reposition einer einfachen Fraktur und Fixierung mittels Schraubenosteosynthese (OPS 5-793.14) führt zur G-DRG I59Z mit einer BWR von 0,775 (2567€). Offene Frakturversorgungen mit anderen Osteosynthesematerialien (konventionelle oder winkelstabile Platten, Drähte/Cerclagen u.a.) bilden sich, sofern keine Materialien kombiniert werden, über die DRG I21Z ab (BWR 0,928; 3074€) ab. Bei einer Kombination von unterschiedlichen Osteosynthesematerialien wird die Basis DRG I31 erreicht, die je nach verwendeter Methode und Art der Fraktur 2-fach differenziert ist. Die Kombination aus Draht- und Schraubenosteosynthese ist der G-DRG I31B zugeordnet (BWR 1,483 $4912 €$ ).

Bei einfachen Frakturen führt eine Kombination aus winkelstabiler Plattenosteosynthese (OPS 5-793.k4) mit zusätzlichen Schrauben- (OPS 5-793.14) und/ oder Drahtosteosynthese (OPS 5-79324) ebenfalls in die I31B. Die gleiche Kombination bei einfachen Frakturen, jedoch durchgeführt mit konventioneller Platte (OPS 5-793.34), führt in die DRG I31A mit einer BWR von 2,253 und einem Erlös von $7462 €$. Die I31A wird ebenfalls bei der oben beschriebenen Kombination und Versorgung einer einfachen Fraktur mit einer Doppelplattenosteosynthese angesteuert, wenn mindestens eine der Platten eine konventionelle Platte ist. Das Gruppierungsergebnis bei Mehrfragmentfrakturen und einer Kombination mehrerer Verfahren ist in Bezug auf die Plattenosteosynthese jedoch dort genau umgekehrt. Die Winkelstabilität von mindestens einer verwendeten Platte (OPS 5-794.k4) führt hier in die I31A, die Verwendung von konventionellen Platten zur Osteosynthese (OPS 5794.24) in die I31B. Die Abb. 5 versucht den hier verwirrenden Gruppierungsmechanismus übersichtlich darzustellen.

Der endoprothetische Ersatz des Radiuskopfs bildet sich über den OPS-Kode 5$824.3 \mathrm{ab}$ und führt in die G-DRG I47A (BWR 2,445 $8098 €$ ). Die Komorbidität der Patienten (PCCL) oder Weichteilschäden sind hierbei für die G-DRG-Gruppierung nicht relevant.

Auch bei der Lokalisation Radiuskopf findet sich eine sehr differenzierte Abbildung der rekonstruktiven/osteosynthetischen Frakturversorgung im G-DRGSystem. Der Implantatkostenanteil bei den G-DRGs I31A und I31B beträgt 11,0 bzw. 9,3\% [6]. Der Implantatkostenanteil der I47A beläuft sich auf 18,5\% [6]. Wird die BWR um diesen Faktor bereinigt, ergeben sich für die G-DRGs I31A und I31B die Werte 2,005 bzw. 1,345, sowie bei der G-DRG I47A 1,993. Während die be- 


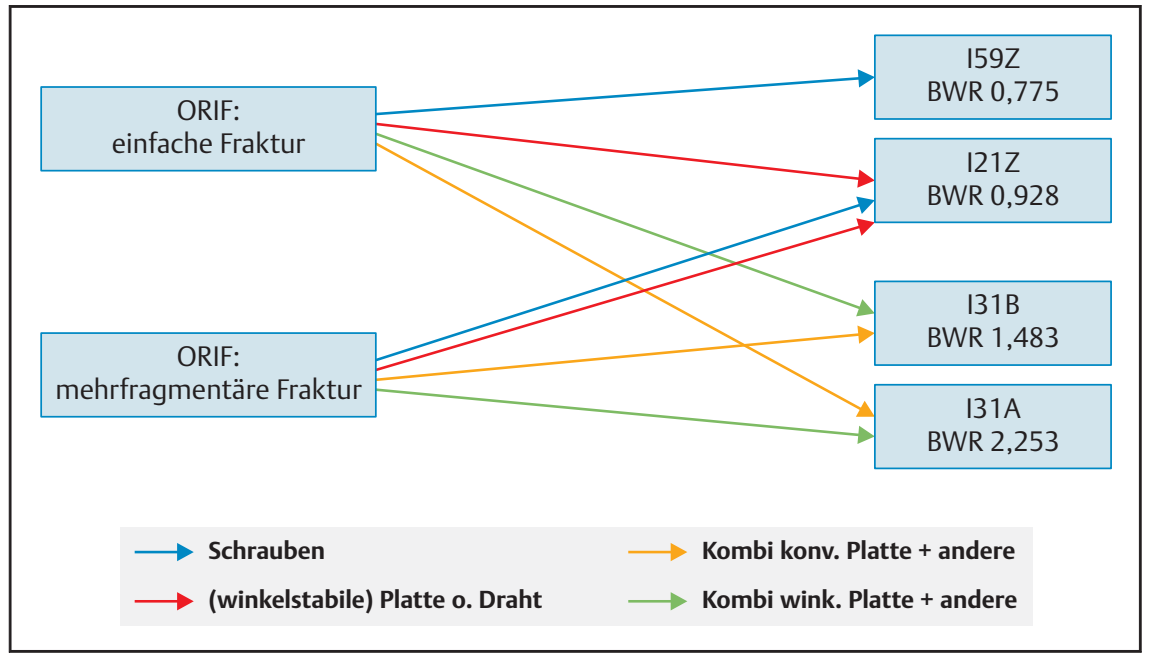

Abb.5 G-DRG-System 2016: osteosynthetische Versorgung einer Radiuskopffraktur durch offene Reposition. Unterschiedliche G-DRG-Zuordnungen insbesondere bei der Versorgung mittels Kombination (Kombi) aus Platten und anderen Osteosyntheseformen (Andere). Verschiedene Ergebnisse bei winkelstabiler und konventioneller Plattenosteosynthese bei Versorgung von einfachen oder mehrfragmentären Frakturen.

reinigten BWR der G-DRGs I31A und I47A vergleichbar sind, zeigt sich ein deutlicher Unterschied zur G-DRG I31B. Dies spiegelt die geringere Komplexität der Eingriffe nur z.T. wider, da es aus klinischer Perspektive nicht ersichtlich ist, warum die Kombination mit konventionellen oder winkelstabilen Platten bei einfachen und mehrfragmentären Frakturen unterschiedlich bewertet wird. Berücksichtigt werden muss hier jedoch, dass die mittlere Verweildauer der DRG I47A bei 11,4 Tagen liegt und damit deutlich höher, als bei den „rekonstruktiven DRGs“ mit 9,2 Tagen (I31A) und 5,0 Tagen (I31B).

\section{Schlussfolgerung}

Es lässt sich zusammenfassen, dass bei allen untersuchten Lokalisationen die rekonstruktiven Eingriffe im G-DRG-System 2016 differenzierter abgebildet sind als die entsprechenden ersetzenden Verfahren. Lediglich im Bereich des Kniegelenks und der Versorgung der Tibiakopffraktur mit endoprothetischem Ersatz gibt es mit 4 G-DRGs (I43A/B und I44A/ $B)$ eine größere Differenzierung. An allen anderen Lokalisationen gibt es einen deutlich höheren Differenzierungsgrad bei den rekonstruktiven Eingriffen, wobei im Bereich der endoprothetischen Versorgung einer Humeruskopffraktur sowie im Bereich des Radiuskopfs jeweils lediglich eine einzige G-DRG angesteuert wird. Die Ursache hierfür wird auch in der deutlichen Ressourcenvarianz der verschiedenen rekonstruktiven Therapien zu sehen sein. Die Zuordnung zu den einzelnen G-DRGs erfolgt an allen Lokalisationen fast ausschließlich über die operativen Prozeduren.

Nebendiagnosen in Form eines hohen PCCL haben, mit Ausnahme der Schenkelhalsfraktur, bei rekonstruktiven Eingriffen keinerlei Auswirkungen auf das Gruppierungsergebnis. Demgegenüber zeigt sich, dass sich ein relevanter Weichteilschaden nicht auf die Gruppierung im Rahmen eines endoprothetischen Ersatzes auswirkt. Da Endoprothesen bei höhergradigem Weichteilschaden aufgrund der erhöhten Infektionsgefahr ohnehin nur selten implantiert werden, folgt dieses Ergebnis auch der klinischen Praxis.

Zusätzlich fällt bei der Analyse aller hier genannten und durch endoprothetische Versorgungen angesteuerten G-DRGs auf, dass sich die Hauptdiagnose „Fraktur" in der untersuchten G-DRG-Systemversion 2016 nicht auf die Gruppierung auswirkt. Die endoprothetische Versorgung bei Fraktur bildet sich genauso ab, wie die „elektive“ Endoprothetik bei degenerativen Erkrankungen. Ausnahme ist hier nur die Fraktur am distalen Femur sowie am Femurschaft, die bei der Implantation einer Kniegelenktotalendoprothese Auswirkungen auf die G-DRG-Gruppierung hat [1]. Die Versorgung einer distalen Femurfraktur durch Rekonstruktion oder endoprothetischen Ersatz wurde in dieser Arbeit nicht genauer analysiert. Diese Entwicklung ändert sich zumindest für die Schenkelhalsfraktur. In der G-DRG-Systemversion
2017 wird die G-DRG-Gruppierung mit Implantation einer Hüftgelenks-TEP entsprechend der Ursache (Koxarthrose vs. Schenkelhalsfraktur) differenziert in 2 G-DRGs abgebildet [2].

Des Weiteren lässt sich feststellen, dass die Bewertungsrelationen der rekonstruktiven Eingriffe deutlich geringer ausfallen als die eines endoprothetischen Ersatzes. Hier muss allerdings beachtet werden, dass die Implantatkostenanteile bei den „endoprothetischen DRGs" deutlich höher ausfallen und einen Teil dieser scheinbar höheren Bewertung ausmachen. Zusätzlich gibt es die Möglichkeit, bei modularen Endoprothesen durch das Zusatzentgelt ZE2016-25 die höheren Implantatkosten auszugleichen. Bei der Betrachtung der um die Implantatkosten bereinigten GDRGs wird die mit dem G-DRG-System angestrebte Umsetzung einer Leistungsorientierung deutlich. Je nach Lokalisation ergeben sich hierbei jedoch unterschiedlich hohe Differenzen, die nur z.T. mit den Erfahrungen der klinischen Praxis korrelieren. Auch die mittleren Verweildauern der "rekonstruktiven DRGs“ im Vergleich zu den „gelenkersetzenden DRGs“ können diese Differenz, insbesondere im Bereich des Hüft- und des Kniegelenks, nicht immer erklären. Zusätzlich sollte in diesem Zusammenhang angemerkt werden, ob die mittleren Verweildauern nicht gerade bei komplexen rekonstruktiven Eingriffen, ggf. mit nachfolgender Teilbelastung einer Extremität, nicht länger als beim entsprechenden endoprothetischen Ersatz sein müssten. Hier müssten differenziertere Analysen, die neben den Implantatkosten auch weitere relevante Kostenanteile berücksichtigen, durchgeführt werden, um weitere Beurteilungen treffen $\mathrm{zu}$ können.

Ein weiterer Faktor, der für das G-DRGSystem 2017 berücksichtigt werden muss, ist die neue Systematik der Sachkostenkorrektur. Diese führt im G-DRGSystem 2017 zu einer Abwertung der Sachkostenanteile und zu einer konsekutiven Aufwertung der Personalkostenanteile einer G-DRG. Dies hat insbesondere Auswirkungen auf G-DRGs mit hohem Sachkostenanteil, wie z.B. in der endoprothetischen Versorgung. Eine genauere Aussage kann hier aber erst nach Veröffentlichung weiterer Informationen durch das InEK getroffen werden, die zum Zeitpunkt der Erstellung des Artikels noch nicht vorlagen. 
Neuere Verfahren, wie z.B. die Zementaugmentation von Schrauben, die im Rahmen der osteosynthetischen Versorgung von Frakturen bei schlechter Knochenqualität zunehmend verwendet werden, sind in der G-DRG-Version 2016 noch nicht gruppierungsrelevant. Entsprechende spezifische OPS-Kodes (586a.3) wurden jedoch erst im OPS 2016 eingeführt, sodass eine Kalkulation der Kosten durch das InEK frühestens für das G-DRG-System 2018 möglich ist. Aus klinischer Perspektive bedarf es auch zukünftig einer engen Abstimmung zwischen Klinikern, der Fachgesellschaft und dem InEK, um die Abbildungsqualität des G-DRG-Systems weiter zu erhöhen, um die Etablierung von Fehlanreizen $\mathrm{zu}$ vermeiden und um eine sachund leistungsgerechte Vergütung orthopädisch-unfallchirurgischer Leistungen sicherzustellen [7].

Interessenkonflikt: Nein.

\section{Literatur}

1 Institut für das Entgeltsystem im Krankenhaus gGmbH. Fallpauschalen-Katalog 2016 (2015). Im Internet: http://www.g-drg.de/cms/GDRGSystem_2016/Fallpauschalen-Katalog/ Fallpauschalen-Katalog_2016; Stand: 04.12.2016

${ }^{2}$ Institut für das Entgeltsystem im Krankenhaus gGmbH. Fallpauschalen-Katalog 2017 (2016). Im Internet: http://www.g-drg.de/cms/GDRGSystem_2017/Fallpauschalen-Katalog2/ Fallpauschalen-Katalog_2017; Stand: 04.12.2016

3 Deutsches Institut für Medizinische Dokumentation und Information (DIMDI). Operationenschlüssel (OPS) Version 2016 (2015). Im Internet: https://www.dimdi.de/static/de/ klassi/ops/kodesuche/onlinefassungen/ opshtml2016/index.htm; Stand: 04.12.2016

4 DIMDI. Internationale statistische Klassifikation der Krankheiten und verwandter Gesundheitsprobleme, 10. Revision, German Modification, (ICD-10-GM), Version 2016 (2015). Im Internet: https://www.dimdi.de/ static/de/klassi/icd-10-gm/kodesuche/ onlinefassungen/htmlgm2016/; Stand: 04.12 .2016

${ }^{5}$ Deutsche Kodierrichtlinien - Allgemeine und Spezielle Kodierrichtlinien für die Verschlüsselung von Krankheiten und Prozeduren,
Version 2016. Im Internet: http://www.gdro.de/cms/G-DRG-System_2016/Kodierrichtlinien/Deutsche_Kodierrichtlinien_2016; Stand: 04.12.2016

${ }^{6}$ Institut für das Entgeltsystem im Krankenhaus gGmbH. G-DRG-Report-Browser 2016 (2015) Im Internet: http://www.g-drg.de/cms/GDRGSystem_2017/Fallpauschalen-Katalog2/ Fallpauschalen-Katalog_2017; Stand: 04.12.2016

${ }^{7}$ Franz D, Schemman F, Selter DD et al. Abbildung orthopädisch-unfallchirurgischer Leistungen in den G-DRG-Systemversionen 2004 und 2014. Unfallchirurg 2014; 117: 946-956

\section{Dr. med. Moritz Freistühler Ärztlicher Mitarbeiter PD Dr. med. Dominik Franz \\ Stv. Geschäftsbereichsleiter}

Geschäftsbereich Medizinisches

Management - Medizincontrolling

DRG-Research-Group

Universitätsklinikum Münster

Niels-Stensen-Straße 8

48149 Münster

Moritz.Freistuehler@ukmuenster.de 